\title{
Classification of helicopter Doppler ambiguous echo signal
}

\section{Czeslaw Lesnik, Piotr Serafin, Adam Kawalec}

Czeslaw Lesnik, Piotr Serafin, Adam Kawalec, "Classification of helicopter Doppler ambiguous echo signal," Proc. SPIE 11442, Radioelectronic Systems Conference 2019, 1144217 (11 February 2020); doi: 10.1117/12.2565722

SPIE. Event: Radioelectronic Systems Conference 2019, 2019, Jachranka, Poland 


\title{
Classification of helicopter Doppler ambiguous echo signal
}

\author{
Czesław Leśnik*a, Piotr Serafin ${ }^{\mathrm{a}}$, Adam Kawalec ${ }^{\mathrm{a}}$ \\ ${ }^{a}$ Military University of Technology, Faculty of Electronics, Institute of Radioelectronics, \\ 00-908 Warszawa, ul. gen. Sylwestra Kaliskiego 2
}

\begin{abstract}
One of the tasks performed by contemporary radars is the classification of a type of detected objects. Among many types of objects, helicopters, and especially hovering ones, are of special interest in radar's self-defence. The echo signal of such object possesses specific properties of its spectrum that result from the construction of the object. A characteristic property of the echo signal is the presence of the spectral components from the hull of the helicopter as well as from the main rotor. The parameters of the latter components depend on the rotary speed of the rotor, the number of the blades and their length.
\end{abstract}

Keywords: helicopter classification, helicopter detection, helicopter blade, helicopter echo signal

\section{INTRODUCTION}

Modern radars, beside detecting objects and estimating their positions, classify the echo signals in order to determine the type of the object. From a tactical point of view it is interesting to distinguish between aircraft with jet propulsion and propeller ones, especially helicopters. The classification criteria exploit kinematical characteristics of the object as well its echo signal properties.

There is a wide range of papers on a helicopter radar echo signal modeling and investigation of its properties in monostatic as well as bi- and multistatic radar systems ${ }^{1-6}$. There are several classification methods considered in the literature, exploiting specific characteristics of the echo signals of such objects ${ }^{7-12}$.

The propeller type aircraft identification feature in practical applications is often implemented as an auxiliary capacity in radars that are optimized for other functions. Therefore there may occur a need for a synthesis of a classification algorithm operating in insufficient, relative to the objects echo Doppler bandwidth, sounding pulse repetition frequency (PRF) conditions. A practical realization of such algorithm requires investigation of subtle properties of the echo signal. In the subsequent sections theoretical considerations of modeling the helicopter's rotor blades and the results of the research on their properties, as well as the results of the examination of the registered real radar signal. A heuristic algorithm for classification of echo signals of propeller driven aircraft is also presented.

\section{HELICOPTER BLADES' ECHO SIGNAL MODELING}

\subsection{Echo signal model}

The echo signal of propeller driven object possesses specific spectral characteristics. The spectrum of such signal contains a component originated by the hull of the helicopter, a component form the hub of the main rotor, a component from the blades of the main rotor and the blades of the tail rotor. Due to the fact, that the tail rotor echo signal has much narrower Doppler bandwidth, this component is often neglected. The following considerations are based on ${ }^{1}$.

The helicopter blades are usually made of metal or composite materials, having a high reflectivity for electromagnetic waves. Let us assume that the rotor is observed by the radar at an elevation angle $\beta=0$ and the azimuth angle $\alpha=0$. The considered rotor contains a single straight blade of a length $L$, mounted on an arm of length $d$. The blade rotates with an angular velocity $\Omega=2 \pi f_{w}$ around a point $O$ located at the distance $R_{0}(t)$ from the radar, where $f_{w}$ is the number of rotates of the blade per second. The geometry of the considered rotor is presented in Fig. 1.

*czeslaw.lesnik@wat.edu.pl; phone+48 26183 7431; fax +48 261837461

Radioelectronic Systems Conference 2019, edited by Piotr Kaniewski, Jan Matuszewski, Proc. of SPIE

Vol. 11442, 1144217 - (c) 2020 SPIE · CCC code: 0277-786X/20/\$21 · doi: 10.1117/12.2565722 
Let us consider a point $P$ located at the initial distance from the radar $R_{P}(t=0)$. The initial distance from the helicopter to the radar is $R_{0}(t=0)=R_{0}$. The initial coordinates of the point $P$ relative to the central point of the rotation $O$ are equal to $P_{0}\left[x_{0}, y_{0}, z_{0}=0\right]$, and the initial angular position of the blade is $\varphi_{0}$.

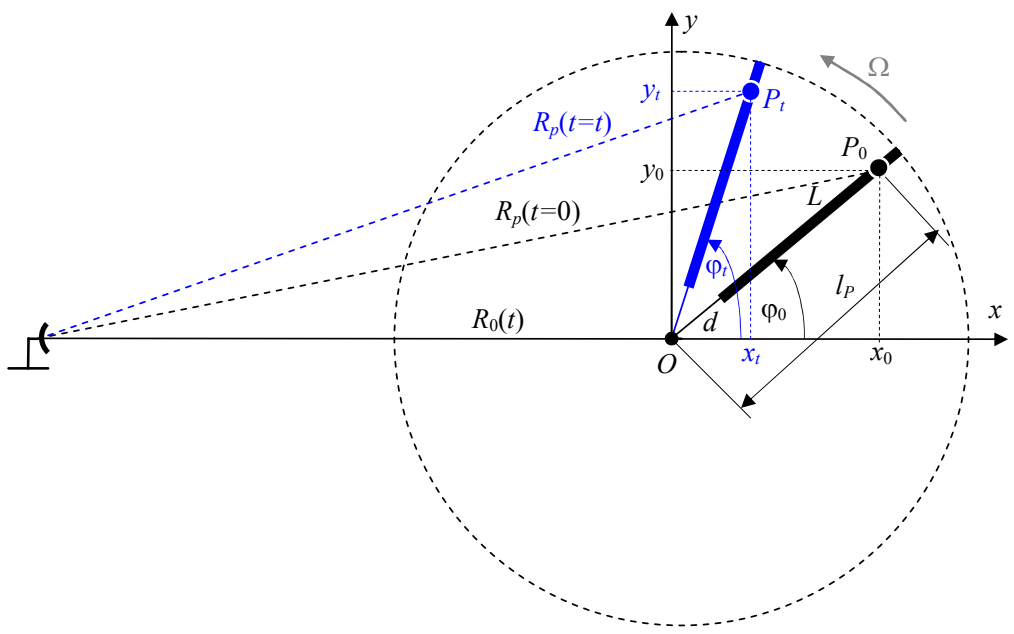

Figure 1. Geometry of the rotor's blade.

After the time period $t$, due to the rotation of the rotor and a non-zero radial component of the velocity $v_{R}$ of the helicopter, located at the initial height $z_{0}$, the distance from the point $P$ to the radar may be expressed as

$$
R_{P}(t) \cong R_{0}+v_{R} t+l_{P} \cos \beta \cos \left(\varphi_{0}+\Omega t\right)+z_{0} \sin \beta .
$$

It can be shown, that for simple (non-modulated) radio pulse with a carrier frequency $f_{t}$, the echo signal form the considered point at the blade, after down-conversion to the baseband and with the constant phase component omitted, takes the form

$$
s_{r}(t)=\exp \left\{-j \frac{4 \pi f_{t}}{c}\left[v_{R} t+l_{P} \cos \beta \cos \left(\varphi_{0}+\Omega t\right)\right]\right\} .
$$

If the length of the arm $d$ is neglected, the combined echo signal from all of the points at the blade can be expressed as

$$
s(t)=\int_{0}^{L} s_{r}\left(t, l_{P}\right) d l_{P} .
$$

In the case of a rotor with $N$ blades, the combined echo signal can be described as

$$
s_{\Sigma}(t)=L \sum_{k=0}^{N-1}\left\{\begin{array}{l}
\left.\exp \left\{-j\left[\frac{4 \pi f_{t}}{c} v_{R} t\right]\right\} \exp \left\{-j \frac{4 \pi f_{t}}{c} \frac{L}{2} \cos \beta \cos \left(\varphi_{0}+\frac{2 \pi}{N} k+\Omega t\right)\right\} \times\right) \\
\times \operatorname{sinc}\left\{\frac{4 \pi f_{t}}{c} \frac{L}{2} \cos \beta \cos \left(\varphi_{0}+\frac{2 \pi}{N} k+\Omega t\right)\right\}
\end{array}\right\} .
$$

\subsection{Investigation on simulated helicopter blade echo signals}

The simulations the research on helicopter blade echo signals has been carried out for a popular Polish "Sokol" helicopter. Its essential parameters take the following values:

- number of the main rotor's blades

- $\quad$ the main rotor diameter
4 ,

$2 \mathrm{~L}=15.7 \mathrm{~m}$, 
- $\quad$ main rotor rotation speed

- number of the tail rotor's blades

- $\quad$ the tail rotor diameter

- tail rotor rotation speed

- maximum speed
$225.7 \mathrm{rpm}$,

2 ,

$2 \mathrm{~L}=3.03 \mathrm{~m}$,

$1342.0 \mathrm{rpm}$,

$100 \mathrm{~m} / \mathrm{s}$.

Basing on (4) the combined echo signal of the all of the main rotor blades for the period one full rotation of the rotor, under the assumption of an exemplary radar pulse repetition interval of $1 \mathrm{~ms}$ and for a helicopter zero radial velocity. Fig. 2a presents this signal normalized to its maximum in values and to the rotation period in time. Fig. $2 \mathrm{~b}$ presents normalized spectrum of this signal against the frequency normalized to the sampling frequency.

(a)

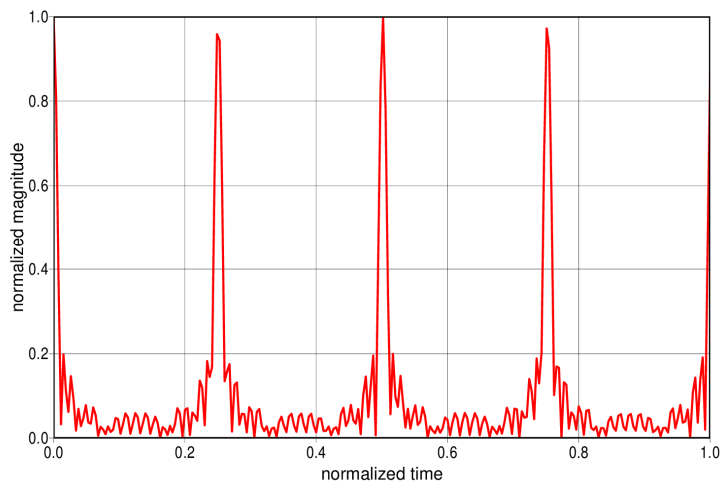

(b)

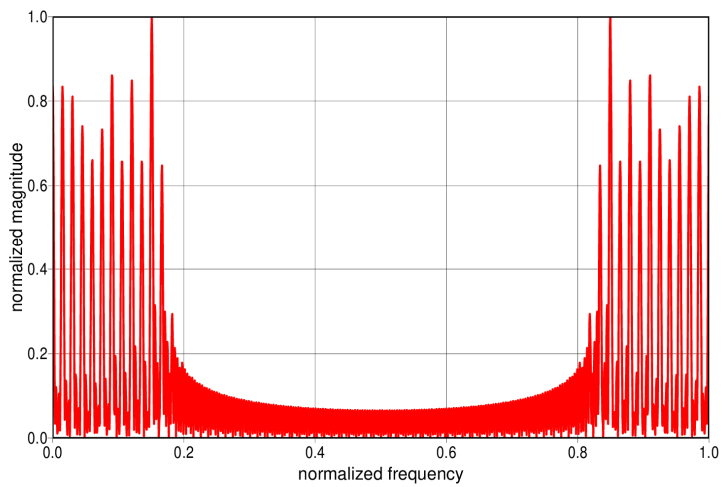

Figure 2. The magnitude of the combined echo signal of the main rotor of the "Sokol" helicopter for $v_{R}=0 \mathrm{~m} / \mathrm{s}$ (a) and the magnitude of its spectrum (b).

Similar characteristics for the assumed maximal radial velocity of $75 \mathrm{~m} / \mathrm{s}$ are presented in Fig. 3 . As the signal in the time domain is presented as the magnitude, the only observable difference is the frequency shift of the magnitude spectrum.

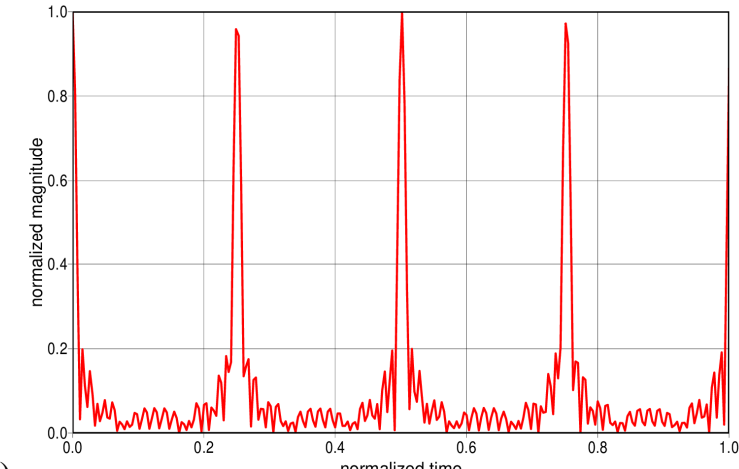

(a)

Figure 3. The magnitude of the combined echo signal of the main rotor of the "Sokol" helicopter for $v_{R}=75 \mathrm{~m} / \mathrm{s}$ (a) and the magnitude of its spectrum (b).

In real radar applications, especially for low pulse repetition frequency radars the Nyquist criterion is often not satisfied, which causes the aliasing effect of the echo signal Doppler spectrum. Fig. 4a shows the magnitude of the helicopter blades echo signal in the case of the Doppler spectrum aliasing, for zero radial velocity. Fig. $4 \mathrm{~b}$ shows its spectrum. 
(a)

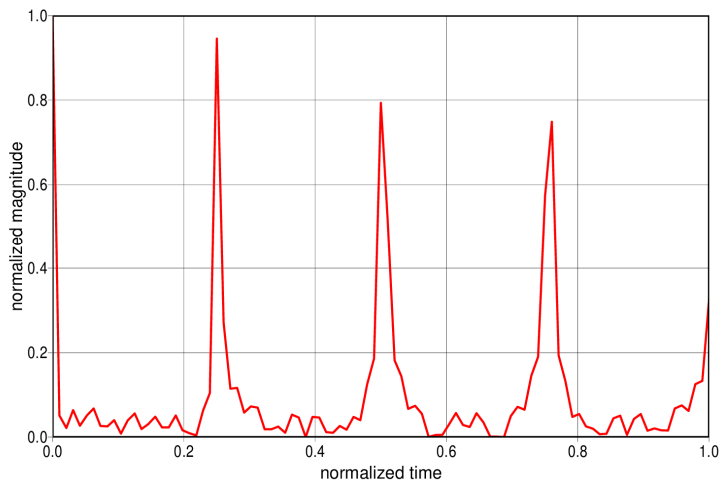

(b)

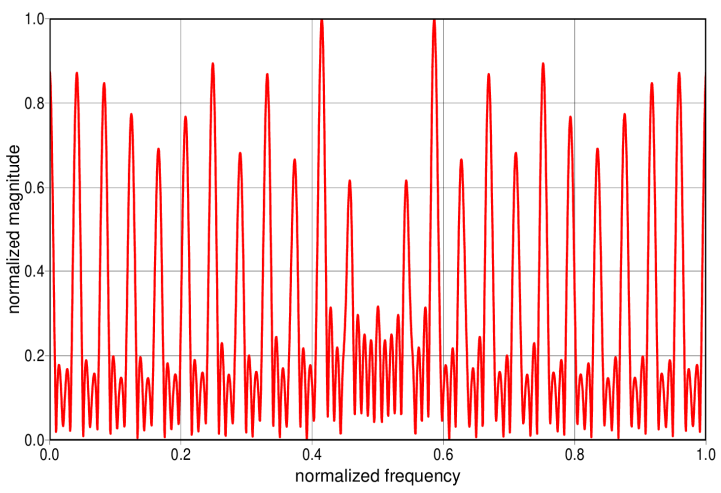

Figure 4. The magnitude of the echo signal of the main rotor of the "Sokol" helicopter for $v_{R}=0 \mathrm{~m} / \mathrm{s}$ (a) and the magnitude of its spectrum (b) in the case of spectrum aliasing.

In Fig. 5 the same characteristics as in Fig. 4 are presented for helicopter radial velocity of $75 \mathrm{~m} / \mathrm{s}$.

(a)

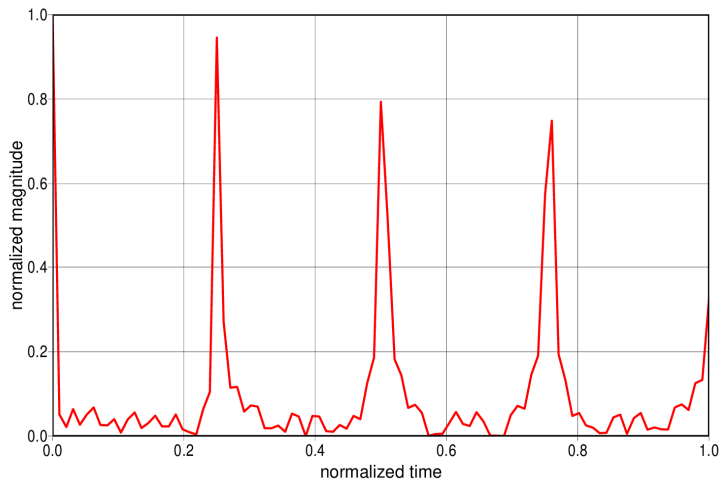

(b)

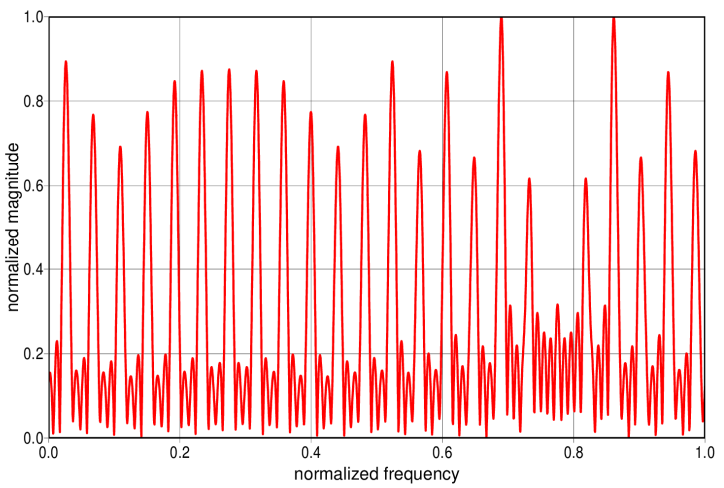

Figure 5. The magnitude of the echo signal of the main rotor of the "Sokol" helicopter for $v_{R}=75 \mathrm{~m} / \mathrm{s}$ (a) and the magnitude of its spectrum (b) in the case of spectrum aliasing.

\section{REAL RADAR SIGNAL ECHO MEASUREMENTS}

A research based on real signals acquired from a low-frequency band search radar has been carried out for different types of aircraft. Figure 6 presents the Doppler spectra of the echo signals from a non-propeller driven object having a nonzero radial velocity. The values of the magnitude of the spectrum are presented in decibels, and the frequency is normalized to the sampling frequency. A characteristic feature of the spectrum is a single maximum at a location dependent on the direction and the value of the radial velocity of the object.

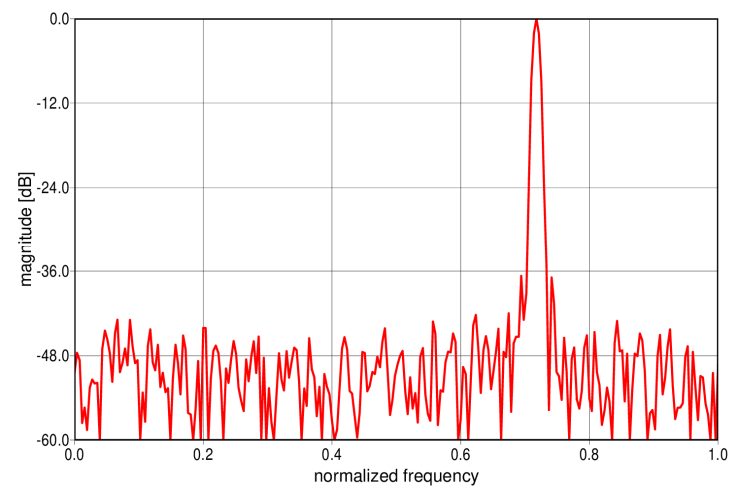

Figure 6. Magnitude of the registered echo signal spectrum a non-propeller driven object. 
Figure 7 presents the magnitude of the Doppler spectrum of the acquired echo signal from two exemplary unknown air objects. The specific shape of the spectrum, containing a sequence of maxima, is typical for echo signals from the rotor's blades. The purpose of the presented on Section 4 algorithm is detection of those multiple maxima being a distinctive feature differing the spectrum of propeller-driven object echo signals from the spectrum of non-propeller-driven object echo signals, presented in Figure 6.

(a)

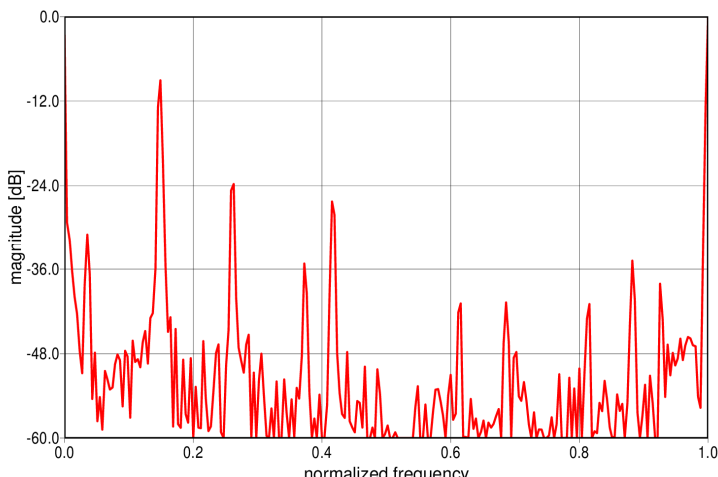

Figure 7. Magnitude of the spectrum of registered echo signals of two (a) and (b) of propeller driven objects.

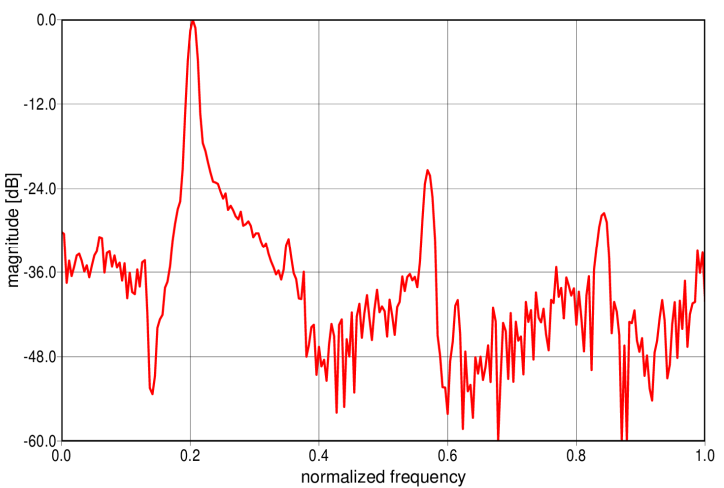

The radar considered in the paper has a low PRF value, which results in the aliasing effect present in the propeller-driven object echo signal spectrum. Due to this an easy to implement analysis of subtle properties of the signal is not possible, and therefore an identification of the type of the detected object, the number of the rotor blades and their length is also not possible. However the spectral analysis of the simulated signals as well as the registered real radar signals indicates differences between the Doppler spectrum of the echo signals from jet driven objects and propeller driven ones.

\section{PROPELLER DRIVEN OBJECTS CLASSIFICATION ALGORITHM}

The above presented differences between the Doppler spectra of the propeller and non-propeller driven object echo signals allowed to develop a relatively simple, practically verified, heuristic algorithm of classification the detected object as a "propeller" or a "non-propeller" one. Its characteristic feature is the ability to identify the propeller-driven object, and especially hovering helicopters, basing on the aliased echo signal spectrum. The ability to detect and classify hovering helicopters is especially desired in military radar systems. The essence of the algorithms may be described as follows.

- Get from the range cell associated with the elementary detection, a slow time samples vector after rangecompression of length equal to the length of the integration window. The length of the integration window is meant as the number of non-coherently integrated pulses of the echo contributing to the elementary detection decision.

- Perform weighting of the samples with a chosen weighting function. The purpose of the weighting of the vector of samples is to lower the level of the side lobes of the echo signal Doppler spectrum. Commonly used weighting functions like Hamming or Blackman-Harris functions were implemented.

- Calculate Fourier transform of the weighted signal, of length allowing for the needed spectral resolution of the analysis. The frequency resolution results both from the properties of the analyzed signal Doppler spectrum, the and the radar technical parameters.

- Perform clutter elimination procedure. Stationary object and clutter echo signal filtration is performed in an Moving Target Indication (MTI) algorithm.

- Calculate the threshold of the analysis. The analysis threshold is an empirically scaled averaged value of the echo signal Doppler spectrum samples.

- Perform a threshold detection of the spectrum components, in the case when a number of $N$ detections is present, where $N$ satisfies the condition $N_{\min } \leq N<N_{\max }$, make a positive identification decision. The value of $N$ is determined on the basis of the difference of the number of maxima in the Doppler spectrum the propeller and nonpropeller-driven object echo signals.

- To each elementary detection, that satisfies the above criterion, set a "propeller" object flag. 
- The final decision on the classification of the object is made at the elementary detections fusion stage, according to the classical $k$ of $n$ rule. The values of $k$ and $n$ parameters depend on the elementary detections' fusion implemented in the radar and are determined empirically.

The parameters of the above algorithm and its correctness were experimentally selected and verified based on a library of registered real radar signals. The, presented in Figure 8, exemplary result of algorithm correctness verification was acquired on the basis of the air object's echo signal, the Doppler spectrum of which is presented in Figure $7 \mathrm{a}$. Additionally the clutter echo signals were present in the analyzed signal.

Figure 8 presents 2-D intensity graphs of magnitude of real radar signals containing aircraft echoes (a) and the results of classification (b).

The horizontal axes are along the range direction, the vertical ones are along the azimuth direction and are scaled in successive pulse repetition intervals. The beginning of the range axes represents zero range, therefore the visible in Fig 8a strong echo signals are from the ground clutter. The propeller driven object echo signal is marked with an oval contour.

(a)

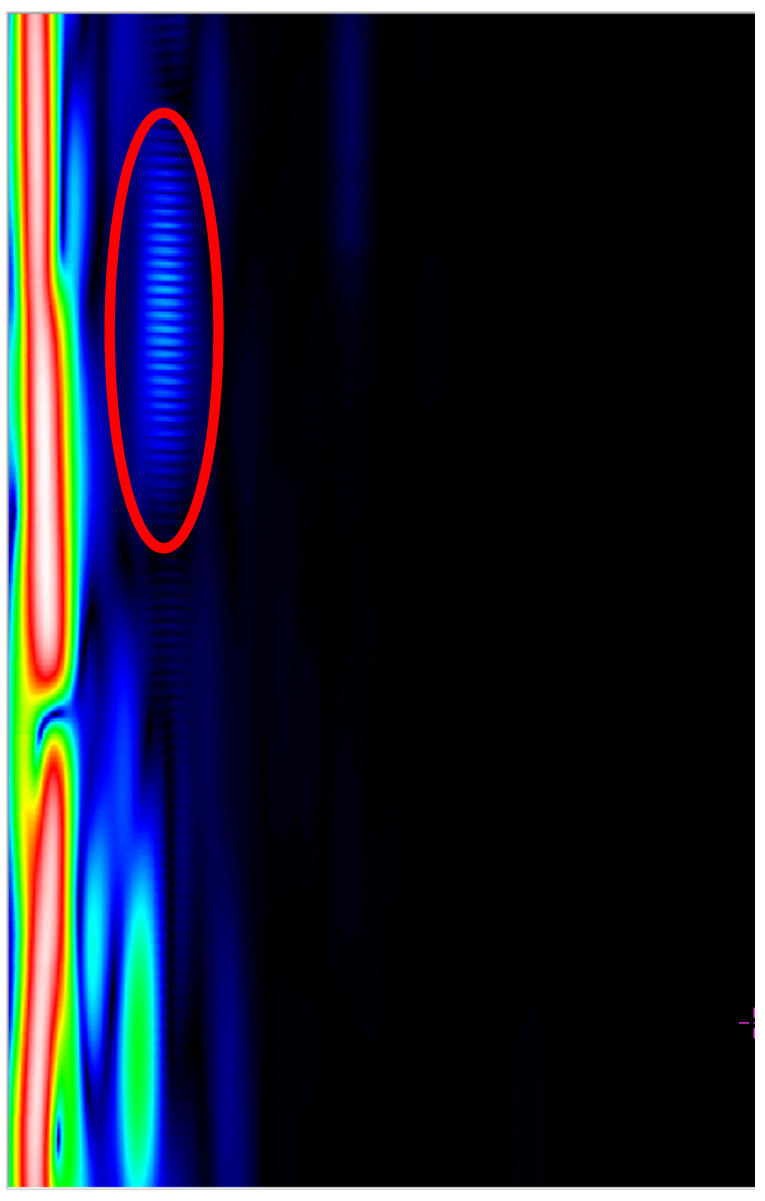

(b)

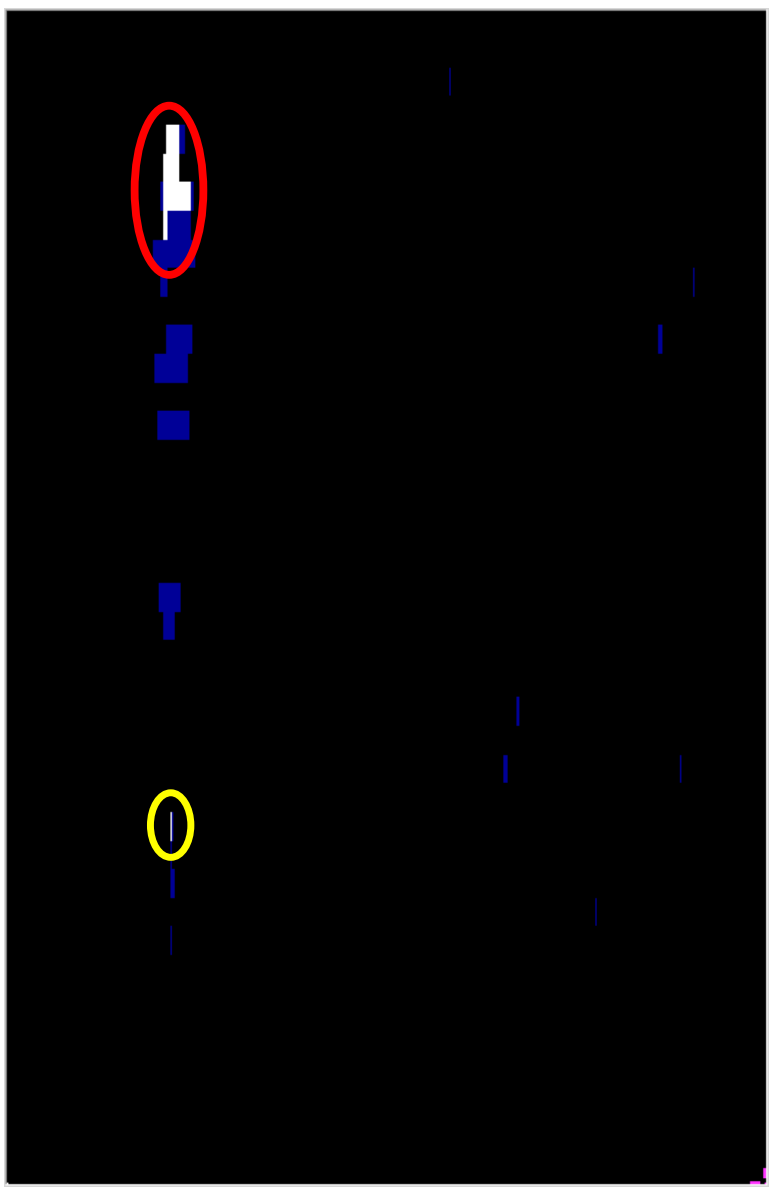

Figure 8. Magnitude of the raw radar echo signals for a selected range - azimuth region (a) and the corresponding object type classification result (b)

In Fig $8 \mathrm{~b}$ shows the results of the classification algorithm. White fields in the graph represent the classification decision "propeller" object, navy blue fields represent the decision to detect a "propeller object" attributed to elementary detections. The red oval contour marks a correct classification of the helicopter while the yellow one marks the false alarm decision that is presented in an explanatory purpose. The size of the false alarm region is rather small, only one range bin. The false alarm decisions are eliminated at the elementary detections fusion stage. 


\section{SUMMARY}

In this paper an analysis of the properties of the propeller driven object radar echo signal, both simulated and registered in radar, have been presented. Its results drove to development of a classification algorithm of such object. The algorithm has been verified with real measurement radar signals. In order to correctly identify the type of the object, the algorithm requires a careful, experimental selection of the parameters.

\section{REFERENCES}

[1] Chen V. C., [The Micro-Doppler Effect in Radar], Artech House, Boston \& London, (2011).

[2] Bączyk, M. K., Krysik, P., Samczyński, P., Misiurewicz, J. and Kulpa, K., "Identification of Helicopter Rotor Parameters using Multistatic Passive Radar", Record of the 2015 IEEE Radar Conference, Arlington, VA, 1464-1467 (2015).

[3] Bączyk, M. K., Misiurewicz, J., Gromek, D. and Kulpa, K., "Analysis of recorded helicopter echo in passive bistatic radar", Proceedings of the $10^{\text {th }}$ European Radar Conference, Nuremberg, Germany, 243-246 (2013).

[4] Kulpa, K., Czekała, Z., Misiurewicz, J. and Falkiewicz. J., "Parametric Detection of the Helicopter Hub Echo", Record of the 1999 IEEE Radar Conference, Waltham, MA, 262-266 (1999).

[5] Burgos-Garcia, M., Perez-Martinez, F. and Gismero Menoyo, J., "Radar Signature of a Long LFM Signal", IEEE Transactions on Aerospace and Electronic Systems, vol. AES-45, no. 3, 1104-1110 (2009).

[6] Misiurewicz, J., Kulpa, K. and Czekała Z., "Analysis of radar echo from a helicopter rotor hub", Record of the 2015 IEEE Radar Conference, Arlington, VA, 1464-1467 (2015).

[7] Zhicheng, Wang et al, "Helicopter Target Recognition Based on the Frequency Domain Adaptive Convolution Kernel Filtering", Record of 2018 China International SAR Symposium (CISS), Shanghai, China (2018).

[8] Burgos-Garcia, M., Perez-Martinez, F. and Gismero Menoyo. J., "Radar Signature of a Long LFM Signal", IEEE Transactions on Aerospace and Electronic Systems, vol. AES-45, no. 3, 1104-1110 (2009).

[9] Misiurewicz, J., Kulpa, K., Czekała, Z. and Filipek. T., "Radar Detection of Helicopters with Application of CLEAN Method", IEEE Transactions on Aerospace and Electronic Systems, vol. AES-48, no. 4, 3525-3537 (2012).

[10] Tang Ziyue, Wang Yongliang, Wu Zhiwen, "STAP scheme to detection of hovering helicopter", Record of the 2000 5th International Conference on Signal Processing Proceedings, Beijing, China, 1921-1924 (2002).

[11]Zhiping Lin, "Detection of helicopter signals using cyclostationarity", Record of the 1995 International Conference on Acoustics, Speech, and Signal Processing, Detroit, MI, USA, 1952-1955 (1995).

[12] Rotander, C. E. and Von Sydow, H., "Classification of helicopters by the L/N-quotient", Record of the RADAR 97, Edinburgh, UK, 629-633 (1997). 